verhalten vorliegen, wenn die schwerste berufsgerichtliche Maßnahme auch in den Fällen ausgesprochen wird, in denen es zu keiner strafrechtlichen Verurteilung gekommen ist. Der Besch. verkennt indes, dass für die allein maßgebliche Prüfung, ob der Berufsangehörige durch sein Verhalten nicht mehr das für die Ausübung seines Berufs unabdingbare Ansehen und Vertrauen besitzt, rechtlich unerheblich ist, ob eine strafrechtliche Verurteilung erfolgt oder wegen Eintritts der Verfolgungsverjährung unterblieben ist.

Der Besch. macht schließlich im Berufungsverfahren erneut geltend, dass eine strafrechtliche Verfolgung seines Verhaltens gemäß $\int 174$ c Abs. 2 StGB wegen des Eintritts der Verjährung nicht mehr möglich sei. Im Hinblick hierauf dürfe auch keine Berufsunwürdigkeit mehr festgestellt werden. Diese Schlussfolgerung ist rechtlich nicht zutreffend.

Die Verfolgungsverjährung für die Straftat war hier zwar gem. os 78 Abs. 3 Nr. 4, 78a StGB i.d.F. v. 27.12.2003 fünf Jahren nach Beendigung der Straftat eingetreten. Der Missbrauch des Behandlungsverhältnisses durch eine sexuelle Handlung an einer Person, die zur therapeutischen Behandlung anvertraut ist, war hier mit der Beendigung des Behandlungsverhältnisses am 31.12.2004 beendet, sodass die Verjährung im Jahre 2009 eintrat. Dieser Umstand hat indes für die Feststellung der Berufsunwürdigkeit des Besch. im heilberufsgerichtlichen Verfahren keine rechtliche Auswirkung. Denn das Vertrauen der Bevölkerung in die Integrität und Zuverlässigkeit des Besch. als Psychotherapeut besteht nicht allein deshalb fort, weil die Zeugin die beruflichen Verstöße des Besch. erst im Oktober 2014 angezeigt hat und bis zum Ablauf der strafrechtlichen Verfolgungsverjährung keine Strafverfolgungsmaßnahmen mehr eingeleitet werden konnten.

Nach $\$ 50$ Abs. 5 S. 2 HeilbG ist in den Fällen des $\$ 50$ Abs. 1 Nr. 5 HeilBG die rechtskräftige Entscheidung öffentlich bekanntzumachen. Die Art der Bekanntmachung ist gemäß $\$ 50$ Abs. $5 \mathrm{~S}$. 3 HeilBG in dieser Entscheidung $\mathrm{zu}$ bestimmen. Da das Berufungsgericht mit dem vorliegenden Urt. lediglich über die Berufung des Besch. zu entscheiden hat, kann es die im erstinstanzlichen Urt. unterbliebene Anordnung nicht selbst treffen. Dies folgt aus dem gemäß $₫ 85 \mathrm{~S}$. 1 HeilbG entsprechend anzuwendenden Verschlechterungsverbot des $\$ 331$ Abs. 1 StPO, nach dem auch die Verschärfung der Bekanntmachungsbefugnis unzulässig ist (vgl. Meyer-Großner/Schmitt, Strafprozessordnung, 59. Aufl. 2016, \331, Rdnr. 21; Rautenberg, in: Gercke/ Julius/Temming u.a., Strafprozessordnung, 5. Aufl. 2017, \331, Rdnr. 19)

[...]

Das Urt. ist unanfechtbar. Ein Rechtsmittel gegen die Entscheidung des Landesberufsgerichts ist in den Regelungen zur Berufsgerichtsbarkeit in $\int \delta 49 \mathrm{ff}$. HeilbG nicht vorgesehen.

https://doi.org/10.1007/s00350-020-5494-2

\section{Anmerkung zu Hess. VGH, Urt. v. 23.8.2018 - 25 A 1027/17.B (VG Gießen)}

\section{Marco Eicher}

Unwürdigkeit zur Ausübung eines Heilberufs liegt nach ständiger Rechtsprechung dann vor, wenn ein Angehöriger der Heilberufe durch ein Verhalten in der Vergangenheit nicht mehr das zur Ausübung des Berufs erforderliche

Rechtsanwalt Marco Eicher,

Fachanwalt für Strafrecht, Fachanwalt für Medizinrecht, Heberer Rechtsanwälte,

Paul-Hösch-Straße 25a, 81243 München, Deutschland
Ansehen und Vertrauen besitzt, das für die Ausübung des Berufs des jeweiligen Heilberufsangehörigen unabdingbar nötig ist. Dies ist im Hinblick auf den in den ärztlichen Berufsordnungen geregelten Vertrauensgrundsatz gem. $\$ 2$ Abs. 2 S. $1 \mathrm{MBO}$ auch für Ärzte relevant. Der Begriff der Unwürdigkeit findet sich in $\$ 2$ Abs. $1 \mathrm{Nr}$. 3 PsychThG (gleichlautend auch $\$ 3$ Abs. 1 Nr. 2 BÄO), wonach etwa eine Approbation auf Antrag (nur) zu erteilen ist, wenn sich der Antragsteller u.a. nicht eines Verhaltens schuldig gemacht hat, aus dem sich seine Unwürdigkeit (oder Unzuverlässigkeit) zur Ausübung des Berufs ergibt.

Im Hinblick auf den Grundsatz der Verhältnismäßigkeit kann jedoch nicht bereits jedes ,schlichte“ Fehlverhalten die Annahme der Unwürdigkeit rechtfertigen. Bei der Beurteilung des Begriffes Unwürdigkeit kommt es darauf an, ob ein schwerwiegendes Fehlverhalten des Heilberufsangehörigen vorliegt, welches bei Würdigung aller Umstände die weitere Berufsausübung zum maßgeblichen Zeitpunkt der letzten Verwaltungsentscheidung als untragbar erscheinen lässt (so BVerwG v. 28.1.2003 zur Unwürdigkeit gem. BÄO, Az. 3 B 149.02, Rdnr. 4, juris; BVerwG v. 27.10.2010, Az. 3 B 61.10, Rdnr. 8, juris; BayVGH vom 22.7.2014, Az. 21 B 14.463, Rdnr. 25, juris; BayVGH vom 28.6.2017, Az. 21 B 16.2065, Rdnr. 16, juris).

Die Aufnahme sexueller Beziehungen zu einer Patientin, welche zudem noch vor Volljährigkeit der Patientin begonnen hat, stellt grundsätzlich nicht nur einen Straftatbestand gem. $\int 174$ c Abs. 2 StGB dar, sondern auch eine schwerwiegende Verletzung des berufsrechtlichen Abstinenzgebots gem. $\$ 13$ Abs. 3, 4 BO. Die Verletzung dieser Patientenschutzvorschriften wiegt nach Auffassung des Gerichts so schwer, dass weder der lange Zeitraum zwischen der letzten Normverletzung und der gerichtlichen Entscheidung von 12 Jahren, noch die seither beanstandungsfreie weitere beruflichen Tätigkeit des Besch., noch gar dessen persönliche Lebensumstände wie auch die gravierenden, tatsächlichen Auswirkungen der Approbationsentziehung eine für den Besch. günstigere Beurteilung rechtfertigen.

Das Gericht stellt zudem klar, dass auch die Tatsache, dass eine strafrechtliche Ahndung gem. $\$ 174 \mathrm{c}$ Abs. 2 StGB bislang unterblieben und aufgrund zwischenzeitlich eingetretener Verfolgungsverjährung gem. \$S 78 Abs. 3 Nr. 4, 78a StGB nicht mehr möglich ist, der berufsrechtlichen Einschätzung und Feststellung der Unwürdigkeit nicht entgegensteht.

Dem Besch. bleibt somit allenfalls die (ferne) Perspektive, dass gem. $\$ 49$ Abs. 3 S. 1 Hess. HeilbG nach Ablauf von 10 Jahren ein Antrag auf erneute Approbation gem. \$2 Abs. 1 PsychThG gestellt werden kann.

\section{Kosmetikbehandlung in der Apotheke}

AMG §69, ApBetrO §1a Abs. 11, §4 Abs. 1 Nr. 1a, ApoG §2 Abs. 1 Nr. 6, §21, §4 Abs. 2

Die Durchführung von Kosmetikbehandlungen in den Räumlichkeiten einer Apotheke kann eine apothekenübliche Dienstleistung mit Gesundheitsbezug sein, wenn hierdurch die Aufrechterhaltung der Arzneimittelversorgung der Bevölkerung aufgrund des zeitlichen Umfangs nicht beeinträchtigt wird (Abgrenzung zu VG Minden, Urt. v. 26.11.2011, 7 K 1647/10).

VG Gießen, Urt. v. 25.3.2019- 4 K 3001/18.GI

Eingesandt vom VG Gießen

bearbeitet von Rechtsanwältin Christiane Müller,

D+B Rechtsanwälte Partnerschaft mbB,

Kurfürstendamm 195, 10707 Berlin, Deutschland 